\title{
Ground state criteria in one-dimensional antiferromagnetic Ising model with long range interaction
}

\author{
Cite as: J. Math. Phys. 61, 043301 (2020); doi: 10.1063/5.0001423 \\ Submitted: 18 January 2020 - Accepted: 26 March 2020 • \\ Published Online: 15 April 2020
}

Azer Kerimov ${ }^{\text {a) }}$ (D)

\section{AFFILIATIONS}

Department of Mathematics, Bilkent University, Ankara, Turkey

a) Author to whom correspondence should be addressed: kerimov@fen.bilkent.edu.tr

\begin{abstract}
One-dimensional long-range Ising models with antiferromagnetic, convex pair interactions are investigated. A new criterion characterizing ground states is given. The criterion and a new transformation yield short proofs identifying and characterizing the ground states. The uniqueness of periodic ground states up to shifts is shown.
\end{abstract}

Published under license by AIP Publishing. https://doi.org/10.1063/5.0001423

\section{INTRODUCTION}

We consider the one-dimensional antiferromagnetic Ising model with the Hamiltonian

$$
H(\varphi)=\sum_{x, y \in Z^{1}, x>y} U(x-y) \varphi(x) \varphi(y)-\mu \sum_{x \in Z^{1}} \varphi(x),
$$

where the spin variables $\varphi(x)= \pm 1$ and $\mu \in R$ is a chemical potential. The antiferromagnetic potential $U(\cdot)>0$ satisfies the following conditions:

(1) $U(x+y)+U(x-y)>2 U(x)$ for all $x, y \in Z^{1}, x>y$

(2) $\quad \sum_{x=1}^{\infty} U(x)<\infty$.

The first condition is a convexity condition, which, to a large extent, determines the structure of the set of ground states. The second condition is necessary for the existence of the thermodynamic limit.

The initial studies of model (1) have been made in Refs. 1-3. It was established that if the configuration satisfies Hubbard's criterion, ${ }^{1}$ then it is a periodic ground state for some chemical potential. The configurations satisfying Hubbard's criterion were constructed in Ref. 4. The existence of configurations satisfying Hubbard's criterion ensures that the criterion is also a necessary condition for periodic ground states. It turns out that these periodic ground states are unique up to translations. ${ }^{5}$ Ground states satisfying Hubbard's condition in the context of non-periodic long-range order, instead of periodic long-range order, are recently investigated in Ref. 6 . It is well known that the models with potentials satisfying $\sum_{x \in Z^{+}} x U(x)<\infty$ have a unique Gibbs state. ${ }^{7-9}$ The uniqueness of Gibbs states of (1) for a wider class of potentials was established in Refs. 10-12.

In this paper, we formulate a criterion equivalent to Hubbard's criterion. Particularly, the criterion allows us to present a very short construction of periodic ground states and prove the uniqueness of those periodic ground states of model (1). Surprisingly, the presented new proof of uniqueness does not even use the structure of ground states.

A configuration $\varphi$ is a function $Z^{1} \rightarrow\{0,1\}$. Let a rational number $q / p$ be fixed. The set of all periodic configurations with period $p$ and $\sum_{y=x+1}^{x+p} \varphi(y)=q$ will be denoted by $\Phi_{p, q}^{p e r}$. The density of a configuration is $q / p$. For each $\phi \in \Phi_{p, q}^{p e r}$, the reciprocal density $\eta(\varphi)$ is equal to $p / q$. 
$\eta(\varphi)$ is an average distance between neighboring points with $\varphi(x)=1$. For each $\varphi \in \Phi_{p, q}^{p e r}$,

$$
h(\varphi)=\frac{1}{p} \sum_{y=x+1}^{x+p} \phi(y) \sum_{z>0} U(z) \varphi(y+z)
$$

not depending on $x$, which is the mean energy of $\varphi$.

\section{GROUND STATE CRITERION}

The following definition is useful for the zero temperature phase diagram of model (1).

A configuration $\varphi_{0} \in \Phi_{p, q}^{p e r}$ is a periodic ground state of (1) if

$$
h\left(\varphi_{0}\right)=\min _{\varphi \in \Phi_{p, q}^{p e r}} h(\varphi) .
$$

For each $\varphi$, if $\varphi(x)=1$, we say that there is a particle at $x \in Z^{1}$. Let $\varphi \in \Phi_{p, q}^{p e r}$ and $\varphi(x)=1$. The distance between $x$ and the $i$-th particle on the right of $x$ will be denoted by $r_{i}(x, \varphi)$.

Hubbard's criterion: Let $\varphi \in \Phi_{p, q}^{p e r}$. If for each $x$ with $\varphi(x)=1$ and for each positive integer $i$,

$$
[i \eta] \leq r_{i}(x, \varphi) \leq[i \eta]+1,
$$

then $\varphi$ is a ground state.

Theorem 1 (Ref. 4). For each rational $0<q / p<1$, there exists $\varphi \in \Phi_{p, q}^{p e r}$ satisfying Hubbard's criterion.

The construction of configurations satisfying Hubbard's criterion uses the decomposition of the inverse density $\eta$ into continued fractions. ${ }^{4}$ Hubbard's criterion provides a sufficient condition for the ground state. As soon as a configuration satisfying Hubbard's criterion is constructed, condition (2) also becomes a necessary condition for characterizing ground states. Hubbard's criterion allows us to get an explicit expression of the mean energy $h(\phi)$ in terms of the reciprocal density $\eta(\varphi)$. Since $h(\phi)$ continuously depends on rational values of $\eta(\varphi)$, it can be extended to the whole interval $[0,1]$. It turns out that at each rational $\eta$, the mean energy $h(\eta)$ has a left-hand derivative $\mu_{\eta}^{-}$and a right hand derivative $\mu_{\eta}^{+}$. and the complement of the set $\bigcup_{\eta}\left(\mu_{\eta}^{-}, \mu_{\eta}^{+}\right)$has Lebesgue measure zero. The dependence of the reciprocal density $\eta$ on the chemical potential is characterized by a devil's staircase type function: for $\mu \in\left(\mu_{\eta}^{-}, \mu_{\eta}^{+}\right)$, the reciprocal density of the periodic ground state is $\eta .3 .4$

We say that a configuration $\varphi^{\prime}$ is a shift of a configuration $\varphi$, if $\varphi^{\prime}(x)=\varphi(x+d)$ for all $x \in Z^{1}$ and some integer $d$.

Theorem 2 (Ref. 5). For each rational $0<q / p<1$, the ground state $\varphi \in \Phi_{p, q}^{p e r}$ is unique up to shifts.

For given $\varphi \in \Phi_{p, q}^{p e r}$ and interval $U=[s, t]$ of length $|U|=t-s$, let $\sigma_{U}(\varphi)$ be the total number of particles in $U$,

$$
\sigma_{U}(\varphi)=\sum_{x \in U} \varphi(x)
$$

Definition. The configuration $\varphi$ is said to be nearly uniform, if for two arbitrary intervals, $U$ and $V$ with equal lengths $(|U|=|V|)$,

$$
\left|\sigma_{U}(\varphi)-\sigma_{V}(\varphi)\right| \leq 1
$$

Theorem 3. A configuration $\varphi \in \Phi_{p, q}^{p e r}$ is a ground state if and only if it is nearly uniform.

Proof. In order to prove this theorem, we will show that a configuration satisfies Hubbard's criterion if and only if it is nearly uniform. Suppose that $\varphi \in \Phi_{p, q}^{p e r}$ satisfies Hubbard's criterion. On the contrary, assume that $\varphi$ is not nearly uniform: there are two intervals $U$ and $V$ such that $|U|=|V|$, but $\sigma_{U}(\varphi)=i, \sigma_{V}(\varphi)=j$, and $i-j \geq 2$. Let $x_{1}$ be the rightmost particle among all particles lying strongly on the left of $V$ and $x_{2}$ be the leftmost particle among all particles lying strongly on the right of $V$. Thus, $x_{2}$ is the $j+1$-th particle on the right of $x_{1}$ and $x_{2}-x_{1} \geq|V|+2$. Now, if we apply Hubbard's criterion to the particle $x_{1}$ and $j+1$, then we get

$$
[(j+1) \eta] \leq r_{j+1}\left(x_{1}, \varphi\right) \leq[(j+1) \eta]+1 .
$$

Let $x_{3}$ and $x_{4}$ be the leftmost and rightmost particles, respectively, inside the interval $U$. Now, if we apply Hubbard's criterion to the particle $x_{3}$ and $i=a-1$, then we get 


$$
[(i-1) \eta] \leq r_{i-1}\left(x_{3}, \varphi\right) \leq[(i-1) \eta]+1 .
$$

Since $i-j \geq 2$, we have $i-1 \geq j+1$, and by (3) and (4),

$$
r_{i-1}\left(x_{3}, \varphi\right)-r_{j+1}\left(x_{1}, \varphi\right) \geq-1 .
$$

On the other hand, since $|U|=|V|, r_{i-1}\left(x_{3}, \varphi\right) \leq|U|$, and $r_{j+1}\left(x_{1}, \varphi\right) \geq|V|+2$, we get

$$
r_{i-1}\left(x_{3}, \varphi\right)-r_{j+1}\left(x_{1}, \varphi\right) \leq-2 .
$$

The contradiction between inequalities (5) and (6) shows that $\varphi$ is nearly uniform.

Now, suppose that the configuration $\varphi \in \Phi_{p, q}^{p e r}$ is nearly uniform. Consider $r_{i}\left(x_{1}, \varphi\right)$ and $r_{i}\left(x_{2}, \varphi\right)$ for some $i$ and particles $x_{1}$ and $x_{2}$, and assume that $r_{i}\left(x_{1}, \varphi\right)-r_{i}\left(x_{2}, \varphi\right) \geq 2$. Let $V=\left[x_{2}, x_{2}+r_{i}\left(x_{2}, \varphi\right)\right]$ and $U$ be any subinterval of $\left[x_{1}, x_{1}+r_{i}\left(x_{1}, \varphi\right)\right]$ not including the endpoints $x_{1}$ and $x_{1}+r_{i}\left(x_{1}, \varphi\right)$ of length $r_{i}\left(x_{2}, \varphi\right)$. Then, $|U|=|V|$, but $\left|\sigma_{U}(\varphi)-\sigma_{V}(\varphi)\right| \geq 2$, a contradiction. Thus, for any $x_{1}$ and $x_{2}$,

$$
\left|r_{i}\left(x_{1}, \varphi\right)-r_{i}\left(x_{2}, \varphi\right)\right| \leq 1 \text {. }
$$

Now, suppose that for some $i, x_{1} r_{i}\left(x_{1}, \varphi\right) \geq[i \eta]+2$. Then, by $(7), r_{i}(x, \varphi) \geq[i \eta]+1$ for all $x$. Consider a sequence $x_{1}, x_{2}, \ldots, x_{q}$, where $x_{n+1}=r_{i}\left(x_{n}, \varphi\right)$ for $n=1, \ldots, q-1$. Then, $\sum_{l=1}^{l=q} r_{i}\left(x_{l}, \varphi\right)>([i \eta]+1) q=([i p / q]+1) q \geq i p$. On the other hand, $\sum_{l=1}^{l=q} r_{i}\left(x_{l}, \varphi\right)=i p$, a contradiction. Similarly, we get a contradiction in the case when for some $x_{1} r_{i}(x, \varphi) \leq[i \eta]-1$. Indeed, in this case, again by $(7), r_{i}(x, \varphi) \leq[i \eta]$ for all $x$. Consider a sequence $x_{1}, x_{2}, \ldots, x_{q}$, where $x_{n+1}=r_{i}\left(x_{n}, \varphi\right)$ for $n=1, \ldots, q-1$. Then, $\sum_{l=1}^{l=q} r_{i}\left(x_{l}, \varphi\right)<([i \eta]) q=([i p / q]) q \leq i p$. On the other hand, $\sum_{l=1}^{l=q} r_{i}\left(x_{l}, \varphi\right)=i p$, a contradiction. Thus, (2) and, hence, Theorem 3 is proved.

Now, we investigate the set of nearly uniform configurations. The restriction of a configuration $\varphi$ to interval $[a, b]$ is called a configuration with support $[a, b]$.

Let $\varphi$ be nearly uniform configuration and $x^{\prime} \in Z^{1}$ such that $\varphi\left(x^{\prime}-1\right)=1$ and $\varphi\left(x^{\prime}\right)=0$. We say that $B=\left\{\varphi\left(x^{\prime}\right), \varphi\left(x^{\prime}+1\right), \ldots\right.$, $\left.\varphi\left(x^{\prime}+t-1\right)\right\}$ is a block containing $t$ points if $\varphi\left(x^{\prime}\right)=\varphi\left(x^{\prime}+1\right)=\cdots=\varphi\left(x^{\prime}+t-1\right)=0$ and $\varphi\left(x^{\prime}+t\right)=1$. By Theorem 1 , each block contains either $[\eta]$ or $[\eta]+1$ points. We say that a block is normal or long if it contains $[\eta]$ or $[\eta]+1$ points. Clearly, the restrictions of the configuration $\varphi$ to both $\left[x^{\prime}, \infty\right)$ and $\left(-\infty, x^{\prime}-1\right]$ are constituted by arrayed normal and long blocks. Thus, $\varphi$ is an arrayed collection of blocks. Let $a=a(\phi)$ and $b=b(\varphi)$ be the total number of normal and long blocks in a period of $\varphi$. Since

$$
a+b=q, a \eta+b(\eta+1)=p,
$$

the values of $a$ and $b$ are uniquely determined by $p$ and $q$.

Let us define a transformation $T_{\eta}$ defined on $\Phi_{p, q}^{p e r}$, where $\eta=p / q$. This transformation plays a crucial role in the proof of Theorems 1 and 2. In order to numerate blocks constituted $\varphi$ by numbers $\ldots-2,-1,0,1,2, \ldots$, we assign a number 0 to the block $B_{0}$ containing the origin of $Z_{1}$ and after that numerate all blocks from the right of $B_{0}$ one by one by numbers $1,2, \ldots$ and all blocks from the left of $B_{0}$ one by one by numbers $-1,-2, \ldots$,

Let us define a configuration $\varphi^{\prime}=T_{\eta}(\varphi)$ by

$$
\varphi=\cdots B_{-2}, B_{-1}, B_{0}, B_{1}, B_{2}, \ldots
$$

$$
\varphi^{\prime}(k)= \begin{cases}1 & \text { if } B_{k} \text { is long } \\ 0 & \text { if } B_{k} \text { is normal. }\end{cases}
$$

Thus, in order to obtain $\varphi^{\prime}$, we put $\varphi^{\prime}(0)=0(1)$, if $B_{0}$ is normal (long) and after that replace each long block by 1 and each normal block by 0 . For example, the piece $\{\ldots 000100010001000100010001000010001 \ldots\}$ of $\varphi$ will be transformed to the piece $\{\ldots 00000010 \ldots\}$. Clearly, $\varphi^{\prime} \in \Phi_{a+b, b}^{p e r}$. Let $U$ be any interval constituted by normal and long blocks of $\varphi$. By definition, $T_{\eta}\left(\varphi(U)\right.$ is the restriction of $T_{\eta}$ to $U$. The support of the configuration $T_{\eta}\left(\varphi(U)\right.$, we denote by $T_{\eta}(U)$.

Clearly, the transformation $T_{\eta}$ is not one to one. Let $\varphi^{\prime \prime} \in \Phi_{p, q}^{p e r}$ such that $T_{\eta}\left(\varphi^{\prime \prime}\right)=\varphi^{\prime}$, and for some $n$, the $B_{0}$ block of $\varphi^{\prime \prime}$ is $[0, n]$. We define the function $T_{\eta}^{-1}$ by $T_{\eta}^{-1}\left(\varphi^{\prime}\right)=\varphi^{\prime \prime}$. Let $U$ be any interval constituted by normal and long blocks of $\varphi^{\prime}$. By definition, $T_{\eta}^{-1}(\varphi(U)$ is the restriction of $T_{\eta}^{-1}$ to $U$. The support of the configuration $T_{\eta}^{-1}\left(\varphi(U)\right.$ is denoted by $T_{\eta}^{-1}(U)$. Let $\mid T_{\eta}\left(\varphi(U) \mid\right.$ and $\mid T_{\eta}^{-1}(\varphi(U) \mid$ be the total number of points in configurations $T_{\eta}\left(\varphi(U)\right.$ and $T_{\eta}^{-1}(\varphi(U)$, respectively.

Lemma 1. Suppose that $\varphi \in \Phi_{p, q}^{p e r}$ is nearly uniform. Then, $T_{\eta}(\varphi) \in \Phi_{a+b, b}^{p e r}$ is also nearly uniform.

Proof. On the contrary, suppose that $\varphi$ is nearly uniform, but $\varphi^{\prime}=T_{\eta}(\varphi)$ is not nearly uniform: there are two intervals $U^{\prime}$ and $V$ with $\left|U^{\prime}\right|=|V|$ such that $\sigma_{U^{\prime}}\left(\varphi^{\prime}\right)-\sigma_{V}\left(\varphi^{\prime}\right) \geq 2$. When we shift $U$ by $1, \sigma_{U}$ changes by 0,1 , or -1 . Therefore, there is an interval $U,|U|=|V|$ such that 
$\sigma_{U^{\prime}}\left(T_{\eta}(\varphi)\right)-\sigma_{V}\left(T_{\eta}(\varphi)\right)=2$. Let us consider the pre-images of $\varphi^{\prime}(U)$ and $\varphi^{\prime}(V)$. Clearly, since $|U|=|V|$, we get $\sigma_{T_{\eta}^{-1} U}(\varphi)-\sigma_{T_{\eta}^{-1} V}(\varphi)=0$. On the other hand, since $\varphi^{\prime}(U)$ contains two more particles than $\varphi^{\prime}(V)$, we get $\left|T_{\eta}^{-1}(U)\right|-\left|T_{\eta}^{-1}(V)\right|=2$. Let $x_{1}$ be the rightmost point of $T_{\eta}^{-1}(U)$ ) [by definitions $\varphi^{\prime \prime}\left(x_{1}\right)=1$ ] and $x_{2}$ be the rightmost point lying on the left (outside) of $T_{\eta}^{-1}(V)$ [by definitions $\varphi^{\prime \prime}\left(x_{2}\right)=1$ ]. Now, we have a contradiction: the intervals $W_{1}=T_{\eta}^{-1}(U)-x_{1}$ and $W_{2}=T_{\eta}^{-1}(V) \cup x_{2}$ have equal lengths, but $\sigma_{W_{1}}\left(\varphi^{\prime \prime}\right)-\sigma_{W_{2}}\left(\varphi^{\prime \prime}\right)=2$ stating that $\varphi^{\prime \prime}$, which is just the shift of $\varphi$, is not nearly uniform.

Lemma 2. Suppose that $\varphi \in \Phi_{a+b, b}^{p e r}$ is nearly uniform. Then, $T_{\eta}^{-1}(\varphi) \in \Phi_{p, q}^{p e r}$ is also nearly uniform.

Proof. On the contrary, suppose that $\varphi$ is nearly uniform, but $\varphi^{\prime}=T_{\eta}^{-1}(\varphi)$ is not nearly uniform: there are two intervals $U$ and $V$ with $|U|=|V|$ such that $\sigma_{U}\left(\varphi^{\prime}\right)-\sigma_{V}\left(\varphi^{\prime}\right) \geq 2$. Let $\sigma_{V}\left(\varphi^{\prime}\right)=c$ and $\sigma_{U}\left(\varphi^{\prime}\right) \geq c+2$. Then, $\varphi^{\prime}(U)$ contains at least $c+1$ blocks and one possibly not complete block $0 \ldots 01$ of length at least 1 . Suppose that $d$ of these $c+1$ blocks are long blocks. Then, readily

$$
|U| \geq(c+1)[\eta]+d+1
$$

On the other hand, $\varphi^{\prime}(V)$ contains at most $c$ blocks and possibly one not complete block $0 \ldots 0$ of length at most $[\eta]-1$. Under transformation, $T c+1$ blocks (all except possibly not complete first block $0 \ldots 01)$ of $\varphi^{\prime}(U)$ transfer to the configuration $\varphi\left(W_{1}\right)$ consisting of $c+1$ points. This configuration contains $d$ particles. Under transformation, $T$ at most $c$ blocks ( $c$ blocks if each out of $c$ particles is included into some block) of $\varphi^{\prime}(V)$ transfer to the configuration $\varphi\left(W_{2}\right)$ consisting of $c$ points. Since $\varphi$ is nearly uniform and $\left|W_{1}\right|>\left|W_{2}\right|$, we get that $\varphi\left(W_{2}\right)$ contains at most $d+1$ particles. Therefore, we readily get

$$
|V| \leq c[\eta]+d+1+([\eta]-1)=(c+1)[\eta]+d,
$$

the inequalities (9) and (10) contradict with $|U|=|V|$.

The following corollary readily follows from the definition of $T_{\eta}$.

Corollary 1. $\varphi$ is a shift of $\varphi^{\prime}$, if and only if $T_{\eta}(\varphi)$ is a shift of $T_{\eta}\left(\varphi^{\prime}\right)$.

The transformation $T$ allows us to present alternative and very short proofs of Theorems 1 and 2.

Proof of Theorem 1. We prove the theorem by induction on $p$. The case $p=2$ is obvious. Suppose that Theorem 2 is proved for all $p<n$. Let us prove the existence of ground state $\varphi \in \Phi_{p, q}^{p e r}$. For given $p, q$, let $a$ and $b$ be unique solutions of $(8)$. Since $a+b<p$ by inductive hypothesis, there exists a ground state $\varphi_{1} \in \Phi_{a+b, b}^{\text {per }}$. Then, $\varphi_{1}$ is nearly uniform, and by Lemma 2 , the configuration $\varphi=T_{\eta}^{-1}\left(\varphi_{1}\right)$ is also nearly uniform. Thus, $\varphi$ is a ground state. Done.

Proof of Theorem 2. We prove the theorem by induction on $p$. The case $p=2$ is obvious. Suppose that Theorem 2 is proved for all periods less than $p$. Let $\varphi_{1}, \varphi_{2} \in \Phi_{p, q}^{p e r}$ be two ground states. By Theorem 3, these configurations are nearly uniform. Consider configurations $T_{\eta}\left(\varphi_{1}\right), T_{\eta}\left(\varphi_{2}\right) \in \Phi_{a+b, b}^{p e r}$, where $a$ and $b$ are unique solutions of (8). By Lemma (1), $T_{\eta}\left(\varphi_{1}\right)$ and $T_{\eta}\left(\varphi_{2}\right)$ are nearly uniform and therefore are also ground states. Since $a+b<p$ by the inductive hypothesis, $T_{\eta}\left(\varphi_{2}\right)$ is a shift of $T_{\eta}\left(\varphi_{2}\right)$. Therefore, by Corollary $1, \varphi_{2}$ is a shift of $\varphi_{1}$. Done.

\section{CONCLUDING REMARKS}

In contrast to Hubbard's criterion, the presented criteria do not involve any explicit condition depending on particle density. The employment of similar ground state criteria may be very efficient in the investigation of antiferromagnetic one-dimensional models with a fixed number of clusters or in higher-dimensional models.

\section{ACKNOWLEDGMENTS}

The author would like to thank the referee for valuable suggestions.

\section{REFERENCES}

${ }^{1}$ J. Hubbard, “Generalized Wigner lattices in one dimension and some applications to tetracyanoquinodimethane (TCNQ)-Salts,” Phys. Rev. B 17, 494-505 (1978).

${ }^{2}$ V. L. Pokrovsky and G. V. Uimin, “On the properties of monolayers of absorbed atoms," J. Phys. C: Solid State Phys. 11, 3535-3549 (1978).

${ }^{3}$ P. Bak and R. Bruinsma, “One-dimensional Ising model and the complete devil's staircase," Phys. Rev. Lett. 49, 249-251 (1982).

${ }^{4}$ S. E. Burkov and Ya. G. Sinai, "Phase diagrams of one-dimensional lattice models with long-range antiferromagnetic interaction,” Usp. Mat. Nauk 38, 205-225 (1983).

${ }^{\mathbf{5}}$ A. Kerimov, "On the ground states of one dimensional antiferromagnetic models with long range interaction," Teor. Mat. Fiz. 58, 473-480 (1984).

${ }^{6}$ A. van Enter, H. Koivusalo, and J. Miękisz, “Sturmian ground states in classical lattice-gas models,” J. Stat. Phys. 178, 832-844 (2020). 
${ }^{7}$ R. L. Dobrushin, "Description of a random field by means of conditional probabilities and conditions for its regularity," Teor. Veroyatn. Primen. 18, 201-229 (1968).

${ }^{8}$ R. L. Dobrushin, "The problem of uniqueness of a Gibbsian random field and the problem of phase transitions," Funk. Anal. Pril. 2, 44-57 (1968).

${ }^{9}$ D. Ruelle, "Statistical mechanics of a one-dimensional lattice gas," Commun. Math. Phys. 9, 267-278 (1968),

${ }^{10}$ A. Kerimov, "Absence of phase transitions in one-dimensional antiferromagnetic models with long-range interactions," J. Stat. Phys. 72, 571-620 (1993).

${ }^{11}$ A. Kerimov, "Uniqueness of Gibbs states in one-dimensional antiferromagnetic model with long-range interaction," J. Math. Phys. 40, 4956-4974 (1999).

${ }^{12}$ M. Fannes, P. Vanheuverzwijn, and A. Verbeure, "Energy-entropy inequalities for classical lattice systems," J. Stat. Phys. 29, 547-559 (1982). 